\title{
AN ITERATION FOR FINDING A COMMON RANDOM FIXED POINT
}

\author{
BINAYAK S. CHOUDHURY
}

Received 12 August 2002 and in revised form 7 October 2003

We define a random iteration scheme and consider its convergence to a common random fixed point of two random operators defined on a convex subset of a separable Hilbert space. We also consider the case when the subset is further compact.

\section{Introduction}

The study of random fixed points has been an active area of contemporary research in mathematics. Some of the recent works in this field are noted in $[1,2,3,11]$. In particular, random iteration schemes leading to random fixed points were introduced in [3]. After that, random iterations for finding solutions of random operator equations and fixed points of random operators have been discussed, as, for example, in $[4,5,6,7,13]$. In the present context, we define an iteration scheme for two random operators on a nonempty closed convex subset of a separable Hilbert space and consider its convergence to a common random fixed point of the two random operators. The two random operators satisfy some contractive inequality. Contractive mappings have often been subjects of fixed point studies. For a review of the subject matter, we refer to [12]. The case where the domain is further compact has also been discussed.

We first review the following concepts which are essentials for our study in this paper. These concepts are obtainable in $[1,3,6]$.

Throughout this paper, $\left(\Omega, \sum\right)$ denotes a measurable space and $H$ stands for a separable Hilbert space. $C$ is a nonempty subset of $H$.

A function $f: \Omega \rightarrow C$ is said to be measurable if $f^{-1}(B \cap C) \in \sum$ for every Borel subset $B$ of $H$.

A function $F: \Omega \times C \rightarrow C$ is said to be a random operator if $F(\cdot, x): \Omega \rightarrow C$ is measurable for every $x \in C$.

A measurable function $g: \Omega \rightarrow C$ is said to be a random fixed point of the random operator $F: \Omega \times C \rightarrow C$ if $F(t, g(t))=g(t)$ for all $t \in \Omega$.

The following result was established in [10]. We present this result as a lemma and omit its proof. 
Lemma 1.1 [10]. Let $H$ be a Hilbert space. Then, for any $x, y, z \in H$ and any real $\lambda$, the following equality holds:

$$
\|(1-\lambda) x+\lambda y-z\|^{2}=(1-\lambda)\|x-z\|^{2}+\lambda\|y-z\|^{2}-\lambda(1-\lambda)\|x-y\|^{2} .
$$

We define a random iteration scheme in the following definition.

Definition 1.2 (random iteration scheme). Let $S, T: \Omega \times C \rightarrow C$, where $C$ is a nonempty convex subset of a separable Hilbert space $H$, be two random operators. Let

$$
g_{0}: \Omega \longrightarrow C
$$

be any measurable function. The random iteration scheme is defined as the following sequence of functions:

$$
g_{n+1}(t)=\left(1-\alpha_{n}\right) g_{n}(t)+\alpha_{n} T\left(t, h_{n}(t)\right)
$$

where

$$
\begin{gathered}
h_{n}(t)=\left(1-\beta_{n}\right) g_{n}(t)+\beta_{n} S\left(t, g_{n}(t)\right), \\
0<a<\alpha_{n}<b<1, \quad a, b \text { are constants, } \forall n=0,1,2, \ldots, \\
0<\beta_{n}<1, \quad \forall n=0,1,2, \ldots, \\
\varlimsup_{n \rightarrow \infty} \beta_{n}<1 .
\end{gathered}
$$

The following result was established in [8]. We state the result in the following lemma. Lemma 1.3 [8]. Let $\left\{x_{n}\right\}$ and $\left\{y_{n}\right\}$ be two sequences in a Hilbert space $H$ such that

$$
\left\|x_{n}\right\| \leq 1, \quad\left\|y_{n}\right\| \leq 1, \quad \forall n=0,1,2, \ldots
$$

Let $\left\{c_{n}\right\}$, with $0<a<c_{n}<b<1$, where $a$ and $b$ are fixed constants, be a sequence of real numbers. Then

$$
\lim _{n \rightarrow \infty}\left\|\left(1-c_{n}\right) x_{n}+c_{n} y_{n}\right\|=1
$$

implies

$$
\lim _{n \rightarrow \infty}\left\|x_{n}-y_{n}\right\|=0
$$

In [8], the result was proved for a uniformly convex Banach space. Since Hilbert spaces are particular cases of uniformly convex Banach spaces, the result is also true in Hilbert spaces. We have stated the result in the form of the above lemma only in the context of Hilbert spaces.

Definition 1.4 [6] (modified Tricomi's condition). Two functions $S, T: C \rightarrow C$, where $C$ is any nonempty subset of a Hilbert space $H$, are said to satisfy the modified Tricomi's condition if

$$
S p=T p=p
$$


implies

$$
\|S x-p\| \leq\|x-p\|, \quad\|T x-p\| \leq\|x-p\|, \quad \forall x \in C .
$$

\section{Fixed point results}

In this section, we have two theorems in which we discuss the convergence of the random iteration scheme (Definition 1.2) to a common random fixed point of two random operators which satisfy certain conditions.

We begin with the proof of the following lemma.

Lemma 2.1. Let $S, T: C \rightarrow C$, when $C$ is any subset of a Hilbert space $H$, satisfy

$$
\begin{aligned}
\|S x-T y\| \leq \max \{ & \|x-y\|, \frac{1}{2}(\|x-S x\|+\|y-T y\|), \\
& \left.\frac{1}{2}(\|x-T y\|+\|y-S x\|)\right\}, \quad \forall x, y \in C .
\end{aligned}
$$

Then S and T satisfy the modified Tricomi's condition (Definition 1.4).

Proof. Let $T p=S p=p$. Putting $y=p$ in (2.1),

$$
\begin{aligned}
\|S x-p\| & \leq \max \left\{\|x-p\|, \frac{1}{2}(\|x-S x\|+0), \frac{1}{2}(\|x-p\|+\|S x-p\|)\right\} \\
& \leq \max \left\{\|x-p\|, \frac{1}{2}(\|x-p\|+\|p-S x\|), \frac{1}{2}(\|x-p\|+\|S x-p\|)\right\}, \\
& (\text { since }\|x-S x\| \leq\|x-p\|+\|p-S x\|) .
\end{aligned}
$$

This shows that $\|S x-p\| \leq\|x-p\|$.

Similarly, $\|T x-p\| \leq\|x-p\|$.

This completes the proof of the lemma.

Theorem 2.2. Let $S, T: \Omega \times C \rightarrow C$, where $C$ is a nonempty closed convex subset of a separable Hilbert space $H$, be two continuous random operators which satisfy the following inequality: for all $x, y \in C$ and $t \in \Omega$,

$$
\begin{aligned}
& \|S(t, x)-T(t, y)\| \\
& \quad \leq \max \left\{\|x-y\|, \frac{1}{2}(\|x-S(t, x)\|+\|y-T(t, y)\|), \frac{1}{2}(\|y-S(t, x)\|+\|x-T(t, y)\|)\right\} .
\end{aligned}
$$

Further, it is assumed that there exists a common random fixed point of $S$ and $T$. Then the random iteration scheme (Definition 1.2), if convergent, converges to a common random fixed point of $S$ and $T$. 
Proof. Let $p: \Omega \rightarrow C$ be a common random fixed point of $S$ and $T$. It is observed that (2.3) implies that for fixed $t \in \Omega, S(t, \cdot)$ and $T(t, \cdot)$ satisfy (2.1). Therefore, by Lemma 2.1,

$$
\begin{aligned}
& \|S(t, x)-p(t)\| \leq\|x-p(t)\|, \\
& \|T(t, x)-p(t)\| \leq\|x-p(t)\|
\end{aligned}
$$

for all $x \in C$ and $t \in \Omega$.

For $t \in \Omega$ and $x \in C$,

$$
\begin{aligned}
\| g_{n+1} & (t)-p(t) \|^{2} \\
= & \left\|\left(1-\alpha_{n}\right) g_{n}(t)+\alpha_{n} T\left(t, h_{n}(t)\right)-p(t)\right\|^{2} \quad(\text { by }(1.3)) \\
= & \left(1-\alpha_{n}\right)\left\|g_{n}(t)-p(t)\right\|^{2} \\
& +\alpha_{n}\left\|T\left(t, h_{n}(t)\right)-p(t)\right\|^{2}-\alpha_{n}\left(1-\alpha_{n}\right)\left\|g_{n}(t)-T\left(t, h_{n}(t)\right)\right\|^{2} \quad(\text { by }(1.1)) \\
\leq & \left(1-\alpha_{n}\right)\left\|g_{n}(t)-p(t)\right\|^{2}+\alpha_{n}\left\|h_{n}(t)-p(t)\right\|^{2} \quad(\text { by }(1.5) \text { and }(2.5)) \\
= & \left(1-\alpha_{n}\right)\left\|g_{n}(t)-p(t)\right\|^{2}+\alpha_{n}\left\|\left(1-\beta_{n}\right) g_{n}(t)+\beta_{n} S\left(t, g_{n}(t)\right)-p(t)\right\|^{2} \quad \text { (by (1.4)) } \\
= & \left(1-\alpha_{n}\right)\left\|g_{n}(t)-p(t)\right\|^{2} \\
& +\alpha_{n}\left\{\left(1-\beta_{n}\right)\left\|g_{n}(t)-p(t)\right\|^{2}+\beta_{n}\left\|S\left(t, g_{n}(t)\right)-p(t)\right\|^{2}\right. \\
& \left.\quad-\beta_{n}\left(1-\beta_{n}\right)\left\|g_{n}(t)-S\left(t, g_{n}(t)\right)\right\|^{2}\right\} \quad(\text { by }(1.1)) \\
\leq & \left(1-\alpha_{n}\right)\left\|g_{n}(t)-p(t)\right\|^{2} \\
& +\alpha_{n}\left\{\left(1-\beta_{n}\right)\left\|g_{n}(t)-p(t)\right\|^{2}+\beta_{n}\left\|g_{n}(t)-p(t)\right\|^{2}\right\} \quad(\text { by }(1.6) \text { and }(2.4)),
\end{aligned}
$$

or, for all $t \in \Omega$ and $n=0,1,2, \ldots, S(t, p(t))=T(t, p(t))=p(t)$ implies

$$
\left\|g_{n+1}(t)-p(t)\right\| \leq\left\|g_{n}(t)-p(t)\right\|
$$

Since $H$ is a separable Hilbert space and $S$ and $T$ are continuous random operators, it follows from [9] that $S(t, x(t))$ and $T(t, x(t))$ are both measurable functions whenever $x(t)$ is measurable. From the construction of the random iteration scheme (Definition 1.2) and from the fact that $C$ is convex, it immediately follows that $\left\{g_{n}\right\}$ is a sequence of measurable functions from $\Omega$ to $C$.

For $t \in \Omega$, let

$$
\left\{g_{n}(t)\right\} \longrightarrow g(t) \quad \text { as } n \longrightarrow \infty
$$

Then $g(t)$, being the limit of a sequence of measurable functions, is also measurable. Further, $C$ is closed, which implies that $g$ is a function from $\Omega$ to $C$. If $g(t)=p(t)$ for all $t \in \Omega$, then the theorem is satisfied. Otherwise, let $\Omega^{\prime}$ be the subset of $\Omega$ such that for $t \in \Omega^{\prime}, g(t) \neq p(t)$. 
Then, for $t \in \Omega^{\prime}$, there exists a positive integer $N=N(t)$ such that for all $n>N(t)$, $g_{n}(t) \neq p(t)$. We construct, for $t \in \Omega^{\prime}$ and $n>N(t)$,

$$
\begin{aligned}
& x_{n}(t)=\frac{\left(g_{n}(t)-p(t)\right)}{\left\|g_{n}(t)-p(t)\right\|}, \\
& y_{n}(t)=\frac{T\left(t, h_{n}(t)\right)-p(t)}{\left\|g_{n}(t)-p(t)\right\|} .
\end{aligned}
$$

Then, for all $t \in \Omega^{\prime}, n>N(t)$,

$$
\left\|x_{n}(t)\right\|=1
$$

For all $t \in \Omega^{\prime}, n>N(t)$,

$$
\begin{aligned}
\left\|T\left(t, h_{n}(t)\right)-p(t)\right\| & \leq\left\|h_{n}(t)-p(t)\right\| \quad(\text { by }(2.5)) \\
& =\left\|\left(1-\beta_{n}\right) g_{n}(t)+\beta_{n} S\left(t, g_{n}(t)\right)-p(t)\right\| \quad(\text { by }(1.4)) \\
& \leq\left(1-\beta_{n}\right)\left\|g_{n}(t)-p(t)\right\|+\beta_{n}\left\|S\left(t, g_{n}(t)\right)-p(t)\right\| \\
& \leq\left\|g_{n}(t)-p(t)\right\| \quad(\text { by }(2.4)) .
\end{aligned}
$$

This shows that for all $t \in \Omega^{\prime}$ and $n>N(t)$,

$$
\left\|y_{n}(t)\right\| \leq 1
$$

Also, for all $t \in \Omega^{\prime}, n>N(t)$,

$$
\begin{aligned}
\left(1-\alpha_{n}\right) x_{n}(t)+\alpha_{n} y_{n}(t) & =\frac{\left(1-\alpha_{n}\right) g_{n}(t)+\alpha_{n} T\left(t, h_{n}(t)\right)-p(t)}{\left\|g_{n}(t)-p(t)\right\|} \quad \text { by (2.9) and (2.10)) } \\
& =\frac{g_{n+1}(t)-p(t)}{\left\|g_{n}(t)-p(t)\right\|} \quad(\text { by }(1.3))
\end{aligned}
$$

Therefore, for all $t \in \Omega^{\prime}, n>N(t)$,

$$
\begin{aligned}
& \left\|\left(1-\alpha_{n}\right) x_{n}(t)+\alpha_{n} y_{n}(t)\right\| \\
& \quad=\frac{\left\|g_{n+1}(t)-p(t)\right\|}{\left\|g_{n}(t)-p(t)\right\|} \longrightarrow 1 \quad \text { as } n \longrightarrow \infty\left(\text { since } g_{n}(t) \longrightarrow g(t)(\neq p(t)) \text { as } n \longrightarrow \infty\right) .
\end{aligned}
$$

From (1.5), (2.11), (2.13), and (2.15), by using Lemma 1.3, we obtain for all $t \in \Omega^{\prime}$, $\lim _{n \rightarrow \infty}\left\|x_{n}(t)-y_{n}(t)\right\|=0$, or, for all $t \in \Omega^{\prime}, n>N(t)$,

$$
\frac{\left\|g_{n}(t)-T\left(t, h_{n}(t)\right)\right\|}{\left\|g_{n}(t)-p(t)\right\|} \longrightarrow 0 \quad \text { as } n \longrightarrow \infty
$$

that is, for all $t \in \Omega^{\prime}$,

$$
\lim _{n \rightarrow \infty}\left\|g_{n}(t)-T\left(t, h_{n}(t)\right)\right\|=0
$$


390 An iteration for finding a common random fixed point

or, for all $t \in \Omega^{\prime}$,

$$
\lim _{n \rightarrow \infty} T\left(t, h_{n}(t)\right)=\lim _{n \rightarrow \infty} g_{n}(t)=g(t) .
$$

Again, for all $t \in \Omega^{\prime}$,

$$
\begin{aligned}
& \left\|g_{n}(t)-S\left(t, g_{n}(t)\right)\right\| \\
& \leq\left\|g_{n}(t)-T\left(t, h_{n}(t)\right)\right\|+\left\|T\left(t, h_{n}(t)\right)-S\left(t, g_{n}(t)\right)\right\| \leq\left\|g_{n}(t)-T\left(t, h_{n}(t)\right)\right\| \\
& +\max \left\{\left\|h_{n}(t)-g_{n}(t)\right\|\right. \text {, } \\
& \frac{1}{2}\left(\left\|g_{n}(t)-S\left(t, g_{n}(t)\right)\right\|+\left\|h_{n}(t)-T\left(t, h_{n}(t)\right)\right\|\right), \\
& \left.\frac{1}{2}\left(\left\|h_{n}(t)-S\left(t, g_{n}(t)\right)\right\|+\left\|g_{n}(t)-T\left(t, h_{n}(t)\right)\right\|\right)\right\} \\
& \leq\left\|g_{n}(t)-T\left(t, h_{n}(t)\right)\right\| \\
& +\max \left\{\left\|h_{n}(t)-g_{n}(t)\right\|\right. \text {, } \\
& \frac{1}{2}\left(\left\|g_{n}(t)-S\left(t, g_{n}(t)\right)\right\|\right)+\left\|h_{n}(t)-g_{n}(t)\right\|+\left\|g_{n}(t)-T\left(t, h_{n}(t)\right)\right\|, \\
& \left.\frac{1}{2}\left(\left\|h_{n}(t)-g_{n}(t)\right\|+\left\|g_{n}(t)-S\left(t, g_{n}(t)\right)\right\|\left\|g_{n}(t)-T\left(t, h_{n}(t)\right)\right\|\right)\right\} \\
& \leq\left\|g_{n}(t)-T\left(t, h_{n}(t)\right)\right\| \\
& +\max \left\{\beta_{n}\left\|g_{n}(t)-S\left(t, g_{n}(t)\right)\right\|,\right. \\
& \frac{1}{2}\left(\left(1+\beta_{n}\right)\left\|g_{n}(t)-S\left(t, g_{n}(t)\right)\right\|+\left\|g_{n}(t)-T\left(t, h_{n}(t)\right)\right\|\right), \\
& \left.\frac{1}{2}\left(\left(1+\beta_{n}\right)\left\|g_{n}(t)-S\left(t, g_{n}(t)\right)\right\|+\left\|g_{n}(t)-T\left(t, h_{n}(t)\right)\right\|\right)\right\} \\
& =\frac{1}{2}\left(\left(1+\beta_{n}\right)\left\|g_{n}(t)-S\left(t, g_{n}(t)\right)\right\|+3 / 2 \| g_{n}(t)-T\left(t, h_{n}(t)\right)\right) \|,
\end{aligned}
$$

or, for all $t \in \Omega^{\prime}$,

$$
\left\|g_{n}(t)-S\left(t, g_{n}(t)\right)\right\| \leq \frac{3}{1-\beta_{n}}\left\|g_{n}(t)-T\left(t, g_{n}(t)\right)\right\| .
$$

Making $n \rightarrow \infty$ in the above, and using (1.7) and (2.17), we obtain for all $t \in \Omega^{\prime}$,

$$
\lim _{n \rightarrow \infty}\left\|g_{n}(t)-S\left(t, g_{n}(t)\right)\right\|=0
$$


or, for all $t \in \Omega^{\prime}$,

$$
\lim _{n \rightarrow \infty} S\left(t, g_{n}(t)\right)=\lim _{n \rightarrow \infty} g_{n}(t)=g(t)
$$

For $t \in \Omega^{\prime}$,

$$
\begin{aligned}
\left\|h_{n}(t)-g(t)\right\| & \left.=\left\|\left(1-\beta_{n}\right)\left(g_{n}(t)-g(t)\right)+\beta_{n}\left(S\left(t, g_{n}(t)\right)-g(t)\right)\right\| \quad \text { (by }(1.4)\right) \\
& \leq\left(1-\beta_{n}\right)\left\|g_{n}(t)-g(t)\right\|+\beta_{n}\left\|S\left(t, g_{n}(t)\right)-g(t)\right\| .
\end{aligned}
$$

Using (2.8) and (2.22), we have, by making $n \rightarrow \infty$ in the above inequality, for all $t \in \Omega^{\prime}$,

$$
\lim _{n \rightarrow \infty} h_{n}(t)=g(t) .
$$

For all $t \in \Omega^{\prime}$,

$$
\begin{aligned}
& \|T(t, g(t))-g(t)\| \leq\left\|T(t, g(t))-S\left(t, g_{n}(t)\right)\right\|+\left\|S\left(t, g_{n}(t)\right)-g(t)\right\| \\
& \leq\left\|S\left(t, g_{n}(t)\right)-g(t)\right\| \\
& +\max \left\{\left\|g_{n}(t)-g(t)\right\|\right. \text {, } \\
& \frac{1}{2}\left(\|g(t)-T(t, g(t))\|+\left\|g_{n}(t)-S\left(t, g_{n}(t)\right)\right\|\right), \\
& \left.\frac{1}{2}\left(\left\|g(t)-S\left(t, g_{n}(t)\right)\right\|+\left\|g_{n}(t)-T(t, g(t))\right\|\right)\right\} .
\end{aligned}
$$

Making $n \rightarrow \infty$ in the above inequality, we have by virtue of (2.8) and (2.22), for all $t \in \Omega^{\prime}$,

$$
\|T(t, g(t))-g(t)\|=0,
$$

or, for all $t \in \Omega^{\prime}$,

$$
T(t, g(t))=g(t) .
$$

From the symmetry of (2.3), we similarly obtain, for all $t \in \Omega^{\prime}$,

$$
S(t, g(t))=g(t)
$$

If $t \in \Omega-\Omega^{\prime}$, then $g(t)=p(t)$, where $p(t)$ is a common random fixed point of $S$ and $T$. Thus (2.27) and (2.28) are also satisfied for $t \in \Omega-\Omega^{\prime}$ and hence for all $t \in \Omega$.

Since $g$ is a measurable function (being the limit of a sequence of measurable functions), it follows that $g: \Omega \rightarrow C$ is a common random fixed point of $S$ and $T$.

Theorem 2.3. If, in Theorem 2.2, the subset $C$ of $H$ is further compact, then the random iteration scheme (Definition 1.2) converges to a common random fixed point of $S$ and $T$.

Proof. Following the same procedure as in Theorem 2.2, we obtain (2.7), (2.17), and (2.21). Since $C$ is compact, for each $t \in \Omega$, there exists $\left\{g_{n(s)}(t)\right\} \subset\left\{g_{n}(t)\right\}$ such that $\left\{g_{n(s)}(t)\right\}$ is convergent. This choice of $\left\{g_{n(s)}(t)\right\}$ depends on $t$; for different values of $t$, the choice of the subsequence may be different. 
392 An iteration for finding a common random fixed point

Let, for $t \in \Omega$,

$$
\lim _{s \rightarrow \infty} g_{n(s)}(t)=k(t)
$$

From the above, it does not follow that $k(t)$ is a measurable function. Afterwards we will prove it to be so.

By (2.17) and (2.21), for $t \in \Omega$,

$$
\lim _{s \rightarrow \infty} T\left(t, g_{n(s)}(t)\right)=\lim _{s \rightarrow \infty} S\left(t, g_{n(s)}(t)\right)=k(t) .
$$

Then, for $t \in \Omega$,

$$
\begin{aligned}
\|T(t, k(t))-k(t)\| \leq & \left\|T(t, k(t))-S\left(t, g_{n(s)}(t)\right)\right\|+\left\|S\left(t, g_{n(s)}(t)\right)-k(t)\right\| \\
\leq & \left\|S\left(t, g_{n(s)}(t)\right)-k(t)\right\| \\
& +\max \left\{\left\|g_{n(s)}(t)-k(t)\right\|,\right. \\
& \frac{1}{2}\left(\|k(t)-T(t, k(t))\|+\left\|g_{n(s)}(t)-S\left(t, g_{n(s)}(t)\right)\right\|\right), \\
& \left.\frac{1}{2}\left(\left\|k(t)-S\left(t, g_{n(s)}(t)\right)\right\|+\left\|g_{n(s)}(t)-T(t, k(t))\right\|\right)\right\} .
\end{aligned}
$$

Making $s \rightarrow \infty$ in the above inequality, and using (2.29) and (2.30), we obtain, for $t \in \Omega$,

$$
\|T(t, k(t))-k(t)\|=0
$$

or, for $t \in \Omega$,

$$
T(t, k(t))=k(t)
$$

In an exactly similar way we prove that for $t \in \Omega$,

$$
S(t, k(t))=k(t)
$$

Thus, for $t \in \Omega, k(t)$ is a common fixed point of $S(t, \cdot)$ and $T(t, \cdot)$.

Next we show that $g_{n}(t) \rightarrow k(t)$ as $n \rightarrow \infty$ for all $t \in \Omega$. Then, by Lemma 2.1, for all $t \in \Omega$,

$$
\begin{gathered}
\left\|S\left(t, g_{n}(t)\right)-k(t)\right\| \leq\left\|g_{n}(t)-k(t)\right\|, \\
\left\|T\left(t, g_{n}(t)\right)-k(t)\right\| \leq\left\|g_{n}(t)-k(t)\right\| .
\end{gathered}
$$


Then, for all $t \in \Omega$,

$$
\begin{aligned}
& \left\|g_{n+1}(t)-k(t)\right\| \\
& \quad=\left\|\left(1-\alpha_{n}\right) g_{n}(t)+\alpha_{n} T\left(t, h_{n}(t)\right)-k(t)\right\| \quad(\text { by }(1.3)) \\
& \quad \leq\left(1-\alpha_{n}\right)\left\|g_{n}(t)-k(t)\right\|+\alpha_{n}\left\|T\left(t, h_{n}(t)\right)-k(t)\right\| \\
& \quad \leq\left(1-\alpha_{n}\right)\left\|g_{n}(t)-k(t)\right\|+\alpha_{n}\left\|h_{n}(t)-k(t)\right\| \quad(\text { by }(2.36)) \\
& \quad=\left(1-\alpha_{n}\right)\left\|g_{n}(t)-k(t)\right\|+\alpha_{n}\left\|\left(1-\beta_{n}\right) g_{n}(t)+\beta_{n} S\left(t, g_{n}(t)\right)-k(t)\right\| \quad(\text { by }(1.4)) \\
& \quad \leq\left(1-\alpha_{n}\right)\left\|g_{n}(t)-k(t)\right\|+\alpha_{n}\left\{\left(1-\beta_{n}\right)\left\|g_{n}(t)-k(t)\right\|+\beta_{n}\left\|S\left(t, g_{n}(t)\right)-k(t)\right\|\right\} \\
& \quad \leq\left(1-\alpha_{n}\right)\left\|g_{n}(t)-k(t)\right\|+\alpha_{n}\left\{\left(1-\beta_{n}\right)\left\|g_{n}(t)-k(t)\right\|+\beta_{n}\left\|g_{n}(t)-k(t)\right\|\right\} \quad(\text { by }(2.35)),
\end{aligned}
$$

or, for all $t \in \Omega$,

$$
\left\|g_{n+1}(t)-k(t)\right\| \leq\left\|g_{n}(t)-k(t)\right\| .
$$

Formulas (2.29) and (2.38) jointly imply that for all $t \in \Omega$,

$$
\lim _{n \rightarrow \infty} g_{n}(t)=k(t) .
$$

Since for $t \in \Omega,\left\{g_{n}(t)\right\} \subset C$ and $C$ is compact, it follows from (2.39) that

$$
k(t) \in C, \quad \forall t \in \Omega \text {. }
$$

Also, by (2.9), $k(t)$ is the limit of a sequence of measurable functions and hence is itself measurable. Formulas (2.33), (2.34), (2.39) and the above-mentioned fact establish that $k: \Omega \rightarrow C$ is a common random fixed point of the random operators $S$ and $T$ and the random iteration scheme actually converges to $k(t)$.

This completes the proof of the theorem.

In the following theorem we note the corresponding result for the nonrandom case.

Theorem 2.4. Let $S, T: C \rightarrow C$, where $C$ is a nonempty convex subset of a Hilbert space $H$ which is not necessarily separable, satisfy (2.1). Let $S$ and $T$ have at least one common fixed point. Then the sequence $\left\{x_{n}\right\}$ obtained as

$$
\begin{gathered}
x_{0} \in C, \\
x_{n+1}=\left(1-\alpha_{n}\right) x_{n}+\alpha_{n} T\left(\left(1-\beta_{n}\right) x_{n}+\beta_{n} S x_{n}\right),
\end{gathered}
$$

where $0<a<\alpha_{n}<b<1, a, b$ are constants,

$$
\begin{gathered}
n=0,1,2, \ldots, \\
0<\beta_{n}<1, \\
\varlimsup_{n \rightarrow \infty} \beta_{n}<1,
\end{gathered}
$$

if convergent, converges to a common fixed point of $S$ and $T$. If further $C$ is compact, then the sequence actually converges to a common fixed point of $S$ and $T$. 
The proof of the theorem may be obtained by exactly the same procedure followed in the proofs of Theorems 2.2 and 2.3. However, the requirement that $H$ is separable is no longer necessary here. This property was required in the proof of Theorem 2.2 in order to establish that $g_{n}$ is measurable for every $n$. But, in the present case, we do not have to ensure that. Thus, separability of $H$ is not necessary in Theorem 2.4.

Open problem. It is not established whether Theorem 2.2 still holds if the condition that $S$ and $T$ have a common fixed point is weakened to the condition that $S(t, \cdot)$ and $T(t, \cdot)$ have common fixed points for all $t \in \Omega$. The problem may further be investigated.

\section{Acknowledgments}

This work was supported by NBHM Project (48/2/2000-R\&D-II/1317), Department of Atomic Energy, Government of India. The support is gratefully acknowledged.

\section{References}

[1] I. Beg and N. Shahzad, Random fixed points of random multivalued operators on Polish spaces, Nonlinear Anal. 20 (1993), no. 7, 835-847.

[2] Random approximations and random fixed point theorems, J. Appl. Math. Stochastic Anal. 7 (1994), no. 2, 145-150.

[3] B. S. Choudhury, Convergence of a random iteration scheme to a random fixed point, J. Appl. Math. Stochastic Anal. 8 (1995), no. 2, 139-142.

[4] A common unique fixed point theorem for two random operators in Hilbert spaces, Int. J. Math. Math. Sci. 32 (2002), no. 3, 177-182.

[5] _ Random Mann iteration scheme, Appl. Math. Lett. 16 (2003), no. 1, 93-96.

[6] B. S. Choudhury and M. Ray, Convergence of an iteration leading to a solution of a random operator equation, J. Appl. Math. Stochastic Anal. 12 (1999), no. 2, 161-168.

[7] B. S. Choudhury and A. Upadhyay, An iteration leading to random solutions and fixed points of operators, Soochow J. Math. 25 (1999), no. 4, 395-400.

[8] W. G. Dotson Jr., On the Mann iterative process, Trans. Amer. Math. Soc. 149 (1970), 65-73.

[9] C. J. Himmelberg, Measurable relations, Fund. Math. 87 (1975), 53-72.

[10] S. Ishikawa, Fixed points by a new iteration method, Proc. Amer. Math. Soc. 44 (1974), 147-150.

[11] L. Liu, Random approximations and random fixed point theorems in infinite-dimensional Banach spaces, Indian J. Pure Appl. Math. 28 (1997), no. 2, 139-150.

[12] B. E. Rhoades, A comparison of various definitions of contractive mappings, Trans. Amer. Math. Soc. 226 (1977), 257-290.

[13] Iteration to obtain random solutions and fixed points of operators in uniformly convex Banach spaces, Soochow J. Math. 27 (2001), no. 4, 401-404.

Binayak S. Choudhury: Department of Mathematics, Bengal Engineering College (Deemed University), Howrah-711103, West Bengal, India

E-mail address: bsc@math.becs.ac.in 


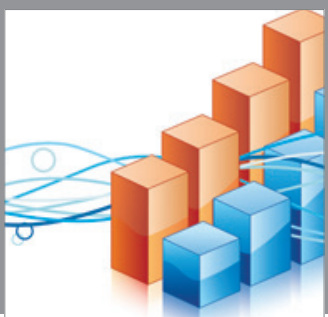

Advances in

Operations Research

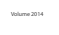

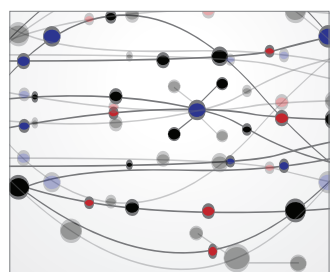

\section{The Scientific} World Journal
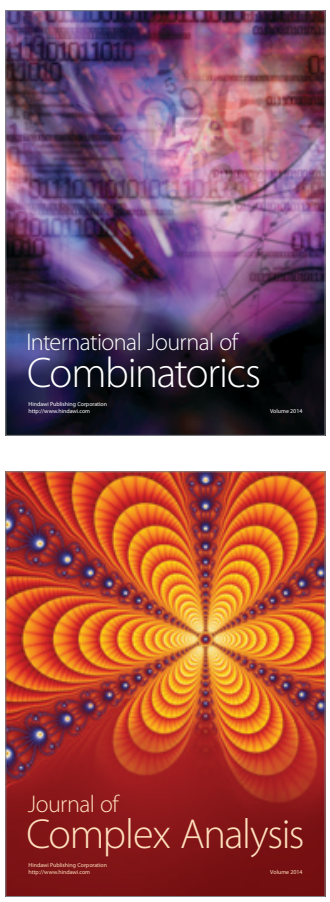

International Journal of

Mathematics and

Mathematical

Sciences
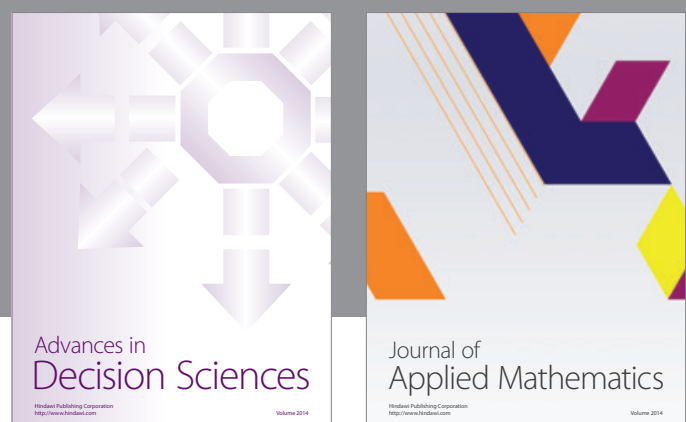

Journal of

Applied Mathematics
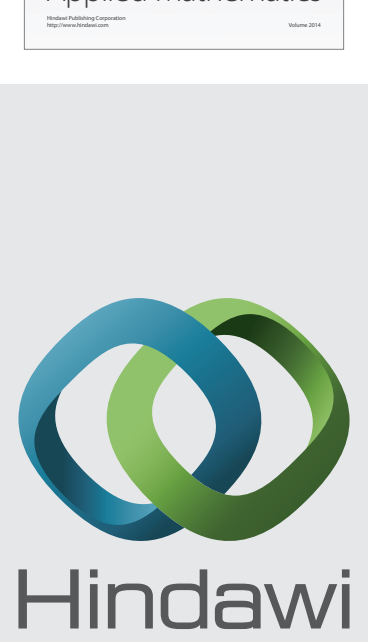

Submit your manuscripts at http://www.hindawi.com
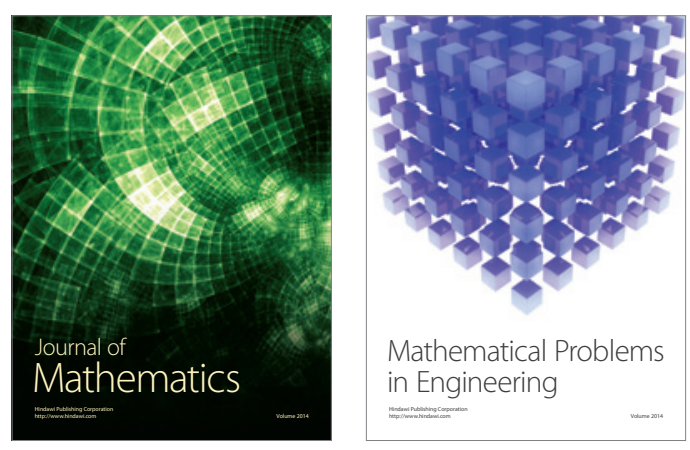

Mathematical Problems in Engineering
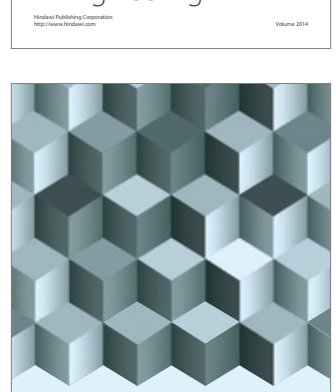

Journal of

Function Spaces
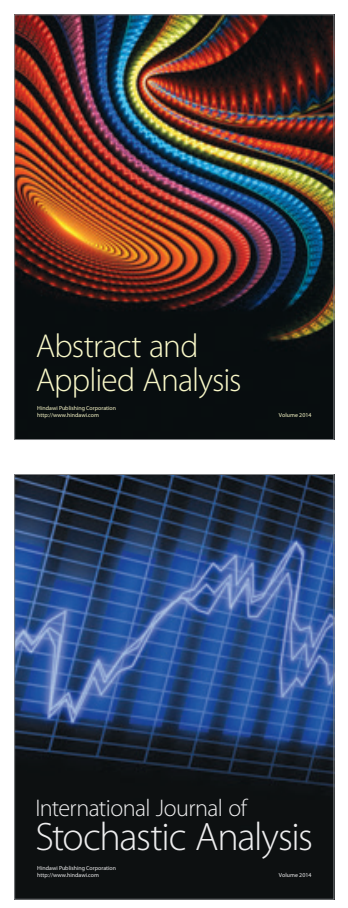

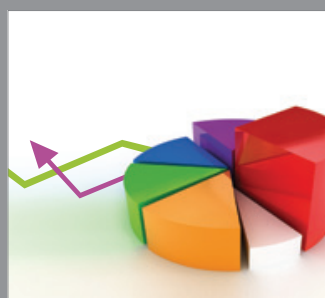

ournal of

Probability and Statistics

Promensencen
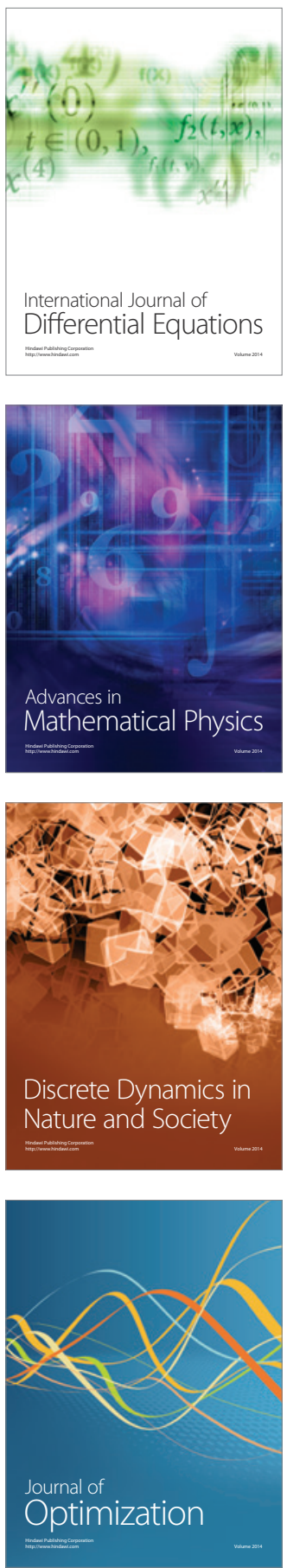